Review Article

\title{
Population Genetical, Behavioural and Evolutionary Studies in the Drosophila bipectinata Species Complex
}

\author{
BASHISTH N SINGH* and PARUL BANERJEE \\ Genetics Laboratory, Department of Zoology, Banaras Hindu University, Varanasi 221 005, India
}

(Received on 07 September 2015; Revised on 14 January 2016; Accepted on 28 January 2016)

\begin{abstract}
Comparison of population dynamics with regard to chromosomal arrangements, protein and nucleotide polymorphism among closely related species can provide invaluable glimpses into the intricacies of speciation. In addition, recently diverged closely related species may also exhibit behavioural differences especially sexual behaviour which can be compared across the species and eventually may aid in understanding the process of speciation. To get the finer details of the so called 'speciation genes', evolutionary studies pertaining to hybrid incompatibilities are required. This involves detailed mapping of the speciation genes through introgression of a candidate gene from one species into the genetic background of another species. Evolutionary studies also include studies on the degree and pattern of sexual isolation among closely related species.

The Drosophila bipectinata species complex comprises four species namely, D. bipectinata, D. parabipectinata, D. malerkotliana and D. pseudoananassae. In three members of this complex i.e. D. bipectinata, D. malerkotliana and $D$. pseudoananassae, subspecies have been identified. This complex with its species and their subspecies is a good model for speciation studies and has been extensively utilized for the purpose. This review attempts to compile all the important population genetical, behavioural and evolutionary studies that have been done in this complex so far. Eventually, we have also discussed what is still left to be done and the prospects of this complex being used in future to get answers relating to speciation.
\end{abstract}

Keywords: Drosophila bipectinata Species Complex; Population Genetical and Behavioural Studies

\section{Introduction}

Studies on the intricacies of speciation involve varied approaches dealing mostly with population genetics, behaviour and evolution.

Population genetics constitutes studies on changes in the genetic composition of a population, spanning investigations on polymorphism at the level of chromosomes (chromosomal arrangements), proteins and nucleotides. Comparing polymorphism levels across different populations of a species throws light on the pattern of polymorphism exhibited by the species. Further, comparing the pattern across different closely related species can definitely indicate the degree of their divergence and phylogenetic relationship.
During the process of speciation, behavioural differentiation is expected as divergence in traits related to male courtship and female preference is necessary for reproductive isolation. Adaptations to their respective niches may also cause differences in certain behavioural aspects in different species. Also, alterations in the behavioural echelon may be a pleiotropic sequel of divergence at a certain locus/ loci not having any direct behavioural implication or simply a by-product of the divergence (although not often the case with behaviour). Therefore, studies on behavioural divergence amongst closely related species aids in unfolding the knots of the complex process of speciation. Behavioural studies mostly involve analysis of the courtship rituals of males and preference patterns of females among closely related

*Author for Correspondence: E-mail: bnsinghbhu@ gmail.com 
species. Apart from these, other aspects of behaviour such as feeding and foraging, nesting, pupation site preference etc. may also be compared among closely related species, demonstrating how differential adaptations aid in speciation.

Evolutionary studies pertaining to the degree and pattern of sexual isolation and genetic basis of hybrid incompatibilities viz sterility, inviability etc. can be very useful in interpreting the evolutionary relationship between a certain species pair. Studies on sexual isolation have also been known to predict the direction of evolution and thereby phylogeny.

While, the approaches to studying speciation are clear, the next important task is the selection of a suitable model organism that would make such studies smoother. Drosophila, which can be easily cultured in the laboratory and has a short generation time, turns out to be very good for speciation studies. Apart from these, the genus also has other advantages that make it the most favoured model for evolutionary and particularly speciation studies. In Drosophila taxonomy, apart from the formally recognised 'species' there also occur 'species groups', 'species subgroups', 'species complexes', 'species clusters' etc. which groups together the closely related species.

Species complexes are important in an evolutionary perspective because the members of a complex while do not hybridize in nature can easily be cross fertilized in the laboratory given no choice situations. This allows novel prospects of undertaking population genetical, behavioural and evolutionary studies leading ultimately to the unearthing of the intricate process of speciation.

The bipectinata complex is one such complex which has been very useful in speciation studies.

\section{Taxonomic characteristics of bipectinata complex}

The melanogaster species group includes a number of subgroups, ananassae subgroup being one of them. Flies belonging to the ananassae subgroup are pale to dark in colour. Males have sex comb in transverse rows on the first two or three tarsal segments and except in D. varians, primary and secondary claspers are present. On the basis of the structure of male genitalia, the ananassae subgroup has been divided into three complexes namely the ananassae complex, the bipectinata complex and the ercepeae complex. While the ananassae complex includes flies with non bifid, apically hirsute aedeagus, bipectinata complex includes flies with bifid, bare and apically hooked adeagus. The erecipae complex on the other hand includes flies with aedeagus having a basiphallus with two plates fused in their basal half and a distiphallus arising from these plates. These complexes are good models for carrying out evolutionary studies as they include flies which have diverged enough to be reproductively isolated but not enough to preclude hybridization (Kopp and Barmina, 2005)

Within the bipectinata complex, we find four species; D. bipectinata (Duda, 1923), D. parabipectinata (Bock, 1971a), D. malerkotliana (Parshad and Paika, 1964) and D. pseudoananassae (Bock, 1971a). The females of all the four species are indistinguishable, but the males can be identified on the basis of intensity of abdominal tip pigmentation and arrangement of sex combs (Figs. 1 and 2). These species share a close phylogenetic relationship and it is postulated that they have diverged not very long back in the evolutionary history i.e. about 283000385000 years ago (Kopp and Barmina, 2005). Though sympatric over parts of their distribution range, they do not hybridize in nature and there is only one report of natural hybridization between $D$. bipectinata and D. malerkotliana (Gupta et al., 1980). However, in the laboratory, given no choice situations, they can be hybridized with varying degrees of crossabilities between each species pair. They are still called good species as consistent with Haldane's rule, among the hybrids, the females are fertile but the males are sterile (Bock, 1971a,b, 1978; Mishra and Singh, 2005a, 2006b; Kopp and Barmina, 2005; Matsuda et al., 2005).

Within three species of this complex subspecies have been identified. Bock (1971a) identified two subspecies each within $D$. malerkotliana and $D$. pseudoananassae. The subspecies of $D$. malerkotliana are called D. malerkotliana malerkotiana and D. malerkotliana pallens and those of $D$. pseudoananassae are called $D$. pseudoananassae pseudoananassae and $D$. pseudoananassae nigrens. This classification is based on the intensity of abdominal tip pigmentation in males (and the subspecies exhibit no differences in sex comb pattern and the structure of male genitalia. D. m. malerkotliana and D. p. nigrens males possess 

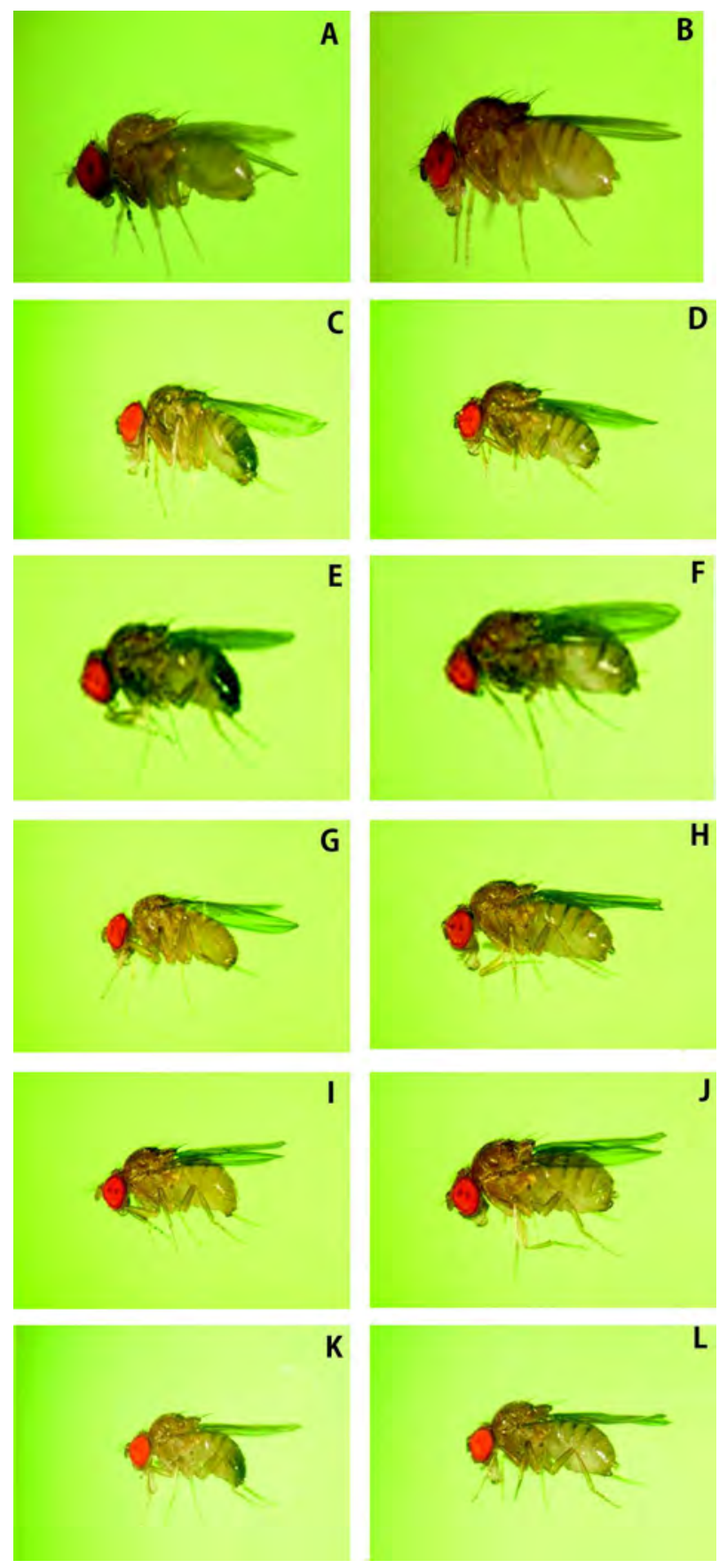

Fig. 1: Males and females of all the species and subspecies of the $D$. bipectinata species complex A. Male of $D$. bipectinata, B. Female of $D$. bipectinata, C. Male of $D$. parabipectinata, D. Female of D. parabipectinata, E. Male of $D$. malerkotliana malerkotliana, F. Female of $D$. malerkotliana malerkotliana, G. Male of $D$. malerkotliana pallens, H. Female of $D$. malerkotliana pallens, I. Male of D. pseudoananassae pseudoananassae, J. Female of $D$. pseudoananassae pseudoananassae, K. Male of $D$. pseudoananassae nigrens, L. Female of D. pseudoananassae nigrens

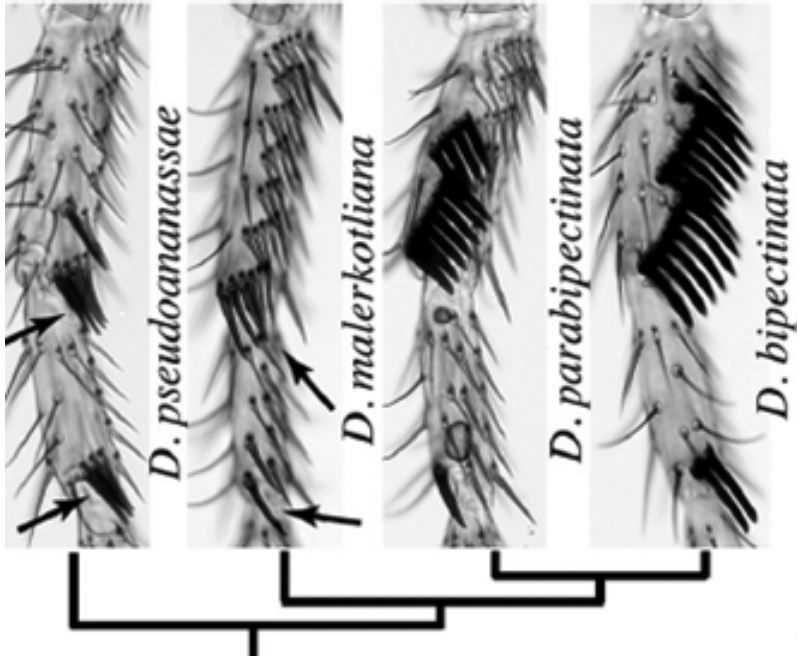

Fig. 2: Sex-comb arrangements in the four species of the bipectinata complex

dark tanned abdominal tip (Fig. 1). There is no evidence of reproductive isolation between the two subspecies of each species. Therefore, though morphologically there is quite a distinct divergence with respect to male abdominal tip pigmentation, behaviourally, there is no difference (Ohtsuka and Tobari, 1976; Aotsuka and Tobari, 1983). Subsequently, Matsuda et al. (2005) identified three subspecies in D. bipectinata, from different geographic locations, based on their studies on reproductive isolation. They are D. bipectinata bipectinata from Southeast Asia (SEA) and Okinawa (OKN), D. bipectinata szentivanii from Papua New Guinea (PNG), and $D$. bipectinata pacificiae from South Pacific Ocean (SPO). The external morphology of the reproductive organs and the number of teeth per row of sex combs were also found to differ significantly between the three subspecies. Kopp and Frank (2005), on the other hand identified two largely allopatric subspecies, Pacific and Asian, exhibiting a 'continuum' of reproductive isolation. Sterile male but fully fertile female hybrids were produced when the two subspecies were crossed. However, no evidence of genetic differentiation could be detected at three randomly chosen nuclear loci, indicating that they are at the earliest stage of speciation (incipient species) and perhaps gene flow is still occurring between them.

Thus, out of the four species in this complex, $D$. parabipectinata is the only one which is not having any subspecies. 
All the four species occur in the OrientalAustralian biogeographic zone, distributed throughout Southeast Asia and extending into Northeastern Australia, the Indian subcontinent and South Pacific. Over parts of this range of distribution, all the four species occur sympatrically (Bock and Wheeler, 1972; Kopp and Barmina, 2005). According to Bock and Wheeler (1972), D. bipectinata is the most widespread species among the four, occurring in India, Southeast Asia and several island groups in the oriental region. However, D. malerkotliana but not $D$. bipectinata has been called a sub cosmopolitan species by Markow and O'Grady (2005). Therefore, both $D$. bipectinata and $D$. malerkotliana are widespread and are expanding their range of distribution. D. pseudoanassae has a fair distribution in India, Southeast Asia and also Australia. D. parabipectinata is having the most restricted distribution, among the four species of this complex.

\section{Population genetical studies in the $D$. bipectinata complex}

Population genetics entails study of the changes taking place in the genetic composition of a population. It can basically be studied at three levels: Chromosomal polymorphism (in Drosophila, presence of polytene chromosomes make that easy), protein polymorphism and nucleotide polymorphism. Population genetical studies have been carried out to a great extent in the species of the Drosophila bipectinata complex.

\section{Chromosomal studies in the complex}

Bock (1971b) studied intra- and interspecific chromosomal inversions in all the four species of the complex. A total of twenty inversions could be detected as extant polymorphisms in these species, Further in the interspecific hybrids ( $F_{1}$ larvae obtained by crossing the two species), twenty autosomal inversions could be detected. In the interspecific hybrids, pairing in polytene chromosomes was found to be excellent in those from crosses involving $D$. bipectinata, $D$. parabipectinata and $D$. malerkotliana but poor in those from crosses involving D. pseudoananassae. Out of these twenty inversions, sixteen were different from those detected in the pure species. From his consummate work in inversions in this complex, Bock constructed a phylogeny whereby $D$. malerkotliana, D. pseudoananassae and a population ancestral to
$D$. bipectinata and D. parabipectinata were said to be derived from a common ancestral population.

Earlier to Bock (1971b), Narda (1968), made first interspecific cross between D. bipectinata and D. maletkotliana and in the $\mathrm{F}_{1}$ larvae, two inversion complexes were found, one in IIL and the other in IIIR. Jha and Rahman $(1972,1973)$, made a reference map of the salivary gland chromosomes of $D$. malerkotliana. They studied the polytene chromosomes of the $\mathrm{F}_{1}$ hybrids of $D$. bipectinata and $D$. malerkotliana and by interpreting the complex configurations of polytene loops, they depicted that the two species differ by seven paracentric inversions, some of which may have been polymorphic in the common ancestral species and the two arrangements (inverted and standard) have become fixed respectively in the two species.

Gupta and Panigrahy (1990), constructed reference polytene map of $D$. bipectinata. They detected nine autosomal paracentric inversions from Indian populations of $D$. bipectinata, distributed on the right and left arms of the second chromosome and the left arm of the third chromosome. Both natural and laboratory populations of $D$. bipectinata were found to be polymorphic due to the presence of three inversions namely In D 2L, In C 2 R and In H 3L. Das and Singh (1992) and Singh and Banerjee (1995) detected three autosomal paracentric inversions from laboratory stocks of $D$. bipectinata, which were found to persist for more than twenty generations in these stocks. Also, there was a significant excess of inversion heterozygotes in all the stocks. Two inversions of the second chromosome namely In D in $2 \mathrm{~L}$ and $\mathrm{In} \mathrm{C}$ in $2 \mathrm{R}$ were found to be associated non randomly exhibiting linkage disequilibrium (Singh and Das, 1991). In natural populations, the frequency of inverted gene orders and the level of inversion heterozygosity were found to be very low as known through the analysis of seven natural populations (Banerjee and Singh, 1996). Also, no evidence for genetic differentiation between populations at the level of inversion polymorphism was found. Therefore, chromosomal polymorphism in D. bipectinata was found to be of the rigid type (Banerjee and Singh, 1996). Populations transferred to laboratory conditions not only remained polymorphic but also there was an increase in the frequency of the inverted gene orders 
due to a rise in inversion heterozygosity (Singh and Banerjee, 1997). Persistence of inversions is indicative of the fact that inversions are maintained by balancing selection in the laboratory populations of $D$. bipectinata. Heterosis in D. bipectinata was found to be due to coadaptation as there was a decline in the frequency of inversion heterozygotes in certain populations derived through interracial hybridizations (Banerjee and Singh, 1998). Singh et al. (2013) also found the In 2RC inversion to persist in seven out of the fifteen laboratory stocks of $D$. bipectinata analysed. A new paracentric inversion was reported in D. malerkotliana from Varanasi by Naseerulla and Hegde (1993 c), which was named as inv IIL C. This was not found by Bock in the D. malerkotliana populations he studied.

Most recently, Tomimura et al. (2005) prepared polytene map of a structurally homozygous stock of D. parabipectinata collected from Brunei, Borneo in1971 and used it as a reference standard sequence for all the four species of the complex. In their extensive study involving various geographical populations of all the species and subspecies of the complex, they could detect as many as 87 inversions in the complex and also described their breakpoints. They also studied polytene of the interspecific hybrids and found that only two arrangements were shared interspecifically. On the basis of the characteristic differences in gene arrangements among the four species they proposed a phylogeny, different from that of Bock's (1971b). According to their phylogeny, $D$. pseudoananassae derives directly from $D$. malerkotliana, from which $D$. bipectinata and $D$. parabipectinata have also been derived.

\section{Studies on Proteins (Isozymes and Allozymes) in the Complex}

Yang et al., 1972, analysed twenty one enzymes corresponding to twenty three loci in all the four members of this complex. Through their study it was concluded that D. bipectinata and D. parabipectinata are extremely similar to each other. Despite this they are able to maintain their distinct identity, existing in sympatry, due to differential niche utilization. According to coefficients of genetic similarity, D. malerkotliana was found to be closely related to D. bipectinata and D. parabipectinata. However, D. pseudoananassae was found to be vastly distant from all the three species. Taking this study further, Hegde and Krishnamurthy, 1976, studied three enzymes (alkaline phosphatase, acid phosphatase and esterase) in the four species and their hybrids. One of the remarkable observations that they made was that the zymograms of hybrids of $D$. bipectinata and $D$. parabipectinata exhibited intermediary banding patterns to the respective parental species. On the other hand, hybrids of $D$. bipectinata and $D$. malerkotliana exhibited a pattern resembling one of the parental species. Studies on alcohol dehydrgenase isozymes in the members of this complex and their hybrids by Jha et al. (1979), revealed that the alleles of Adh are codominantly inherited in the $\mathrm{F}_{1}$ hybrids. They also pointed the role of $\mathrm{X}$ chromosomes on the expression of Adh isozymes in D. bipectinata and D. malerkotliana. The allozymes of acid phosphatase were found to exhibit differences with respect to specific activities, $\mathrm{p}^{\mathrm{h}}$ dependent activity profile, Vmax and $\mathrm{Km}$ values in D. malerkotliana (Parkash, 1993). Allelic frequencies of $A d h$ was found to exhibit clinal variation (Sharma et al., 1993a) and ethanol tolerance was also found to differ latitudinally among $D$. bipectinata populations (Sharma et al., 1993 a, b) and was said to be driven by natural selection (Parkash et al., 1994) indicating rapid divergence among $D$. bipectinata populations. D. malerkotliana populations too exhibit signs of divergence as ethanol utilization indices and ethanol tolerance threshold values in larval and adult individuals were found to vary latitudinally in different Indian populations of $D$. malerkotliana by Sharma et al. (1994). Likewise a number of studies demonstrated that $D$. bipectinata and D. malerkotliana populations (of India) show signatures of divergence among themselves. For example, Naseerulla and Hegde (1993 a, b), compared electrophoretic variation in two enzymes, alkaline phosphatase and glucose 6-phosphate dehydrogenase in D. bipectinata and D. maletkotliana populations collected from microclimatic zones and found that while in D. malerkotliana, the allelic frequencies and heterozygosity per individual for alkaline phosphatase showed significant variation, in D. bipectinata only one allele of the enzyme was found to exhibit interpopulation variation. Glucose 6-phosphate dehydrogenase however exhibited insignificant interpopulation differences in both D. bipectinata and D. malerkotliana (for references see the review by Singh, 2015). 


\section{Nucleotide Divergence Among the Members of the Complex}

Gupta et al. (1993) studied divergence at mt-DNA by analysing the restriction profiles in two members of the complex: D. bipectinata and D. malerkotliana and altogether 8 mitochondrial haplotypes were detected in the total eighteen populations studied (the two species together). Only a single haplotype (IB) was shared by the two species. The net nucleotide substitution per site between the two species was found to be 0.0002 which is hardly any divergence and such a level of difference may even be found among different populations of a species, or between two subspecies. Gupta et al. explained that the two species exhibit poly or paraphyletic relationship in the mt-DNA sequence. These studies were followed by ones directly using the sequence information utilizing the sequence alignment tools. The assembly of $D$. bipectinata genome has been recently generated (Genbank AFFE00000000.1). Kopp and Barmina (2005), combined phylogenetic and population genetic approaches to reconstruct the evolutionary history of the complex, which included reconstruction of the order and timing of speciation events, the extent of genetic differentiation among species and variation within species and the levels of gene flow across species boundaries. For accomplishing this, they used sequences of one mitochondrial and six nuclear loci and all the subspecies known were utilized. Further, Kopp et al. (2006) studied divergence at the Ychromosome in this complex and confirmed the utility of Y- chromosomal loci in construction of phylogeny. From these studies it was concluded that nucleotide divergence among $D$. bipectinata, $D$. parabipectinata and D. malerkotliana is extremely low and they diverged not very long back in the evolutionary time scale. Signor et al. (2013) generated new genomic resources for seven species and subspecies of the $D$. ananassae species group to study intra- and interspecific variation. These genomic resources were transcriptome sequence data. A large number of polymorphic nucleotide positions were identified for each pair of strains. 15245, 343156, 216786 and 318507 single nucleotide polymorphisms were found in D. m. malerkotliana/D. m. pallens, D. m. maletkotliana/D. bipectinata, D. ercepeael $D$. merina and D. p. pseudoananassaelD. pseudoanannassae nigrens respectively. These
SNPs represented approximately 58, 62, 66 and 75\% of all genes in the genome respectively and an average of $46 \%$ of the SNPs was found to be fixed differences. Generation of such transcriptome sequence data not only endorse high- throughput genotyping but can also be used to generate linkage maps rapidly and efficiently thus allowing high resolution genetic analysis.

\section{Behavioural Studies in Four Species of the Complex}

\section{Sexual Behaviour}

Hegde and Krishnamurthy (1979) studied courtship and mating behaviour in the species and subspecies of this complex and found that while courtship behaviour did not vary much within a species (different populations or strains of a species were studied), significant differences were found among the species and the subspecies. Crossley (1986), studied courtship sounds and associated behaviours in the complex. Males of all the four species were found to sing two songs, long and short, during courtship and due to differences in at least one song parameter each species was found to be having a unique song profile. This is thought to have played a very important role in sexual isolation among the members. She also noted that males of all the four species courted females of their own species and those of the other species equally and it was the females which discriminated homospecific from heterospecific. Crossley also described 'female sounds' in this species complex and reported that when bursts of female song were long, they appeared to be inhibitory as male song did not recommence until female song ceased. Also several bursts of female song resulted in breaking up of courtship altogether. This also points that females of this complex are very discriminating. Therefore, courtship rituals of males and preference of females have diverged together to bring about isolation in this complex. From these studies it was also concluded that while $D$. bipectinata, $D$. parabipectinata and D. malerkotliana are closely related to each other, D. pseudoananassae shares a more distant phylogenetic relationship with these three.

Crossley and Bennet-Clark (1993) demonstrated that two male songs when played in a naturally occurring sequence in courtship enhanced female 
receptivity in $D$. parabipectinata, thus corroborating that stimulated courtship songs, has a positive effect on female receptivity.

A number of studies have been performed correlating the courtship and mating patterns with body size of the flies (Ewing and Benet-Clark, 1968; Hegde and Naseerulla, 1992; Naseerulla and Hegde, 1993; Hegde and Krishna, 1997; Krishna and Hegde, 1997). However, in most of these studies either only $D$. malerkotliana was involved or D. bipectinata and D. malerkotliana were involved, since these two species are more widely distributed than the others in the complex. Flies mating first were found to have longer wings and forelegs in D. malerkotliana (Hegde and Naseerulla, 1992), also males with long wings were more successful at courtship and mating (Hegde and Krishna, 1997). That larger flies have a mating advantage was also proven by employing different choice situations in the species $D$. bipectinata and D. malerkotliana (Krishna and Hegde, 1997).

The sexual behaviour of $D$. bipectinata occupies a distinct position in the $D$. bipectinata complex archives since it has been explored to a large extent. Due to genetic heterogeneity among different populations of $D$. bipectinata, mating propensity has been found to differ significantly among them with males showing more variations (Singh and Sisodia, 1995). Similarly courtship time, duration of copulation and fertility have been found to differ significantly among different strains of $D$. bipectinta with duration of copulation also having a positive correlation with fertility (Sisodia and Singh, 1996b). Sexual selection was demonstrated in D. bipectinata through studies on the mating success of interstrain hybrids (Sisodia and Singh, 1996a). Singh and Sisodia (1996a) were also successful at making artificial selection lines (fast and slow) for mating propensities.

Several studies were also performed to test the effect of different mutations on mating activity in $D$. bipectinata. Cut wing mutation was found to have no effect on the sexual activity and neither did it affect sexual isolation in any way (Singh and Sisodia, 1996a). However, mutants having thoracic outgrowth although did not show any difference in mating propensity from their wild type counterparts, did exhibit sexual isolation from wild type and also revealed positive assortative mating. Thus, it was proven that the mutation affects the mate recognition system (Singh and Sisodia, 1996b). Sepia eye colour mutation was found to diminish the sexual activity of $D$. bipectinata males but not the females (Singh and Sisodia, 1999). Singh and Sisodia (1997) also provided evidence for rare male mating advantage in $D$. bipectinata by employing the cut wing mutation.

Kamimura and Polak (2014) studied the intromittent organs of $D$. bipectinata to find out whether surgical manipulations of these organs affect insemination success. There results indicated that spines in these organs are evolving in response to sexual selection, although other explanations could also be given like cryptic female choice.

\section{Effect of Age in Mating Behaviour in Members of this Complex}

Age has a pronounced effect on the mating behaviour and other fitness traits of flies. The optimum age at which maximum reproductive success is achieved varies in different species of Drosophila and may even be different in closely related species. Somashekar et al. (2011), studied female age influence on mating success, courtship activities, mating latency, copulation duration, fecundity, ovariole number and wing length in isofemale lines of $D$. bipectinata collected from three different localities. It was found that middle aged females had greater mating success and were less fussy about the courting males. They also copulated longer and had greater fecundity and more ovarioles than the young and oldaged females. Therefore, the hierarchy of age specific female reproductive success was illustrated as middle aged $>$ young $>$ old aged. While the middle aged females were found to be reproductively superior, the offspring of older males were found to be better in $D$. bipectinata in another study by Krishna et al. (2012). Confirming the good gene model, D. bipectinata females were found to prefer old-aged males. When offspring of old and young aged males were reared in low and high larval densities, it was found that offspring of old aged males had significantly greater pre-adult fitness (egg-larval hatchability and larval-adult viability) and adult fitness (Son's mating success, mating latency, mating ability, progeny production and longevity and daughter's mating success, fecundity and longevity) when reared in both the conditions as 
compared to the progeny of young males. Santosh and Krishna (2013) studied male accessory gland cells (number and size), the quantity of accessory gland proteins and their effect on fitness in males of different ages in D. bipectinata. It was found that $D$. bipectinata young males with smaller sized accessory glands and fewer and larger main cells in the accessory glands produced the least quantity of protein and transferred significantly less protein and sperm to its partner than did the middle and old aged males. Similar to earlier findings they also suggested that females mating with older males obtained a fitness benefit. Similarly, Shivakumar and Krishna (2014) found that middle aged $D$. malerkotliana males remated more than young or old males. They found that females mating with middle aged males which mated only once in an hour were least fecund while female mated with middle aged males mating more than once in an hour produced greater number of progeny.

\section{Analysis of Remating Behaviour}

Studies on remating behaviour have also been carried out extensively in the members of this complex. Remating behaviour is of great evolutionary consequence, especially in the genus Drosophila. Although a Drosophila female gathers enough sperm in the first mating itself to last a lifetime, the phenomenon of remating has been selected in nature because it generates genetic variability. The remating time, duration of copulation in first and second matings and other tenets of this behaviour may vary in different species depending upon their respective priorities. Singh and Singh (2013), studied intra and interspecific variations in female and male remating frequency, latency and duration of copulation in the first and second matings in the members of this complex. Significant intraspecific variations were found in the number of remated females and males in $D$. bipectinata and D. malerkotliana. In $D$. pseudoananassae, while females exhibited significant intraspecific variations males did not. In D. parabipectinata however, variations were found to be insignificant in both males and females with regard to these aspects of remating. The duration of first mating was found to be longest in D. bipectinata and it also took the shortest time to remate. On the other hand, in D. pseudoananassae though the duration of copulation in first mating was long it took the longest time to remate. In all the four species the duration of copulation in second mating was found to be shorter than the duration of copulation in first mating. These differences were explained appropriately and it was concluded that enough sperm are transferred in D. bipectinata in the first mating itself to last a lifetime yet it chooses to mate again so as to maintain genetic variability. The duration of copulation in the first mating remains high because in the face of adversity the luxury of getting more than one partner may not be possible. $D$. pseudoananassae, on the other hand, takes longer to remate as the sperm transferred during first mating are utilized maximally before the female seeks another mate and remating occurs more for the maintenance of population size than generation of variations. In $D$. parabipectinata, the maintenance of population size and genetic variations are equally important and the costs of mating and remating have been adjusted accordingly. It was finally concluded that $D$. bipectinata and $D$. pseudoananassae fall at two extremes and D. parabipectinata and $D$. malerkotliana lie at an intermediate position in the hierarchy of remating behaviours.

Singh and Singh (2014a) also studied the correlation between mating latency, duration of copulation and fertility in four species of the complex. They found significant interspecific variation in mean duration of copulation and fertility in four species of the complex. D. bipectinata was found to show positive correlation between duration of copulation and fertility. D. malerkotliana was found to show negative correlation between mating latency and duration of copulation and $D$. pseudoananassae was found to show positive correlation between mating latency and fertility. Therefore, mating latency, duration of copulation and fertility interact differently in four members of the complex according to the requirements of nature each has to meet in order to thrive successfully in nature. Shivakumar and Krishna (2014) studied male remating in males of different age groups in $D$. malerkotliana. They found that males of all age groups invest (in terms of insemination) more towards the first female than the second. 


\section{Sperm Displacement in Members of this Complex}

Another important aspect of remating behaviour is the occurrence of sperm displacement. Sperm competition in the female reproductive tract, leading to displacement of sperm from the first copulation by the sperm from the second has been recognised as a form of post copulatory sexual selection and the phenomenon, in Drosophila has been well documented by several workers in both natural and laboratory conditions (Singh et al., 2002). Singh and Singh (2014 b, 2015), studied sperm displacement in the complex and came up with very interesting findings. In $D$. bipectinata, the proportion of second male progeny (P2') values were found to range from 0.60 0.67 , indicating that $D$. bipectinata females use sperm from both first and second males after remating but more from the second male. In D. malerkotliana, the $\mathrm{P} 2$ ' values ranged from $0.67-0.70$. However, since the duration of copulation in first mating is less in $D$. malerkotliana as compared to D. bipectinata, it was postulated that there are fewer sperm left in $D$. malerkotliana female than in $D$. bipectinata female when she goes for remating, therefore the P2' values are more in D. malerkotliana. In this way, taking into account the amount of sperm that gets transferred during the first mating, sperm competition in $D$. malerkotliana is less intense as compared to $D$. bipectinata. In $D$. parabipectinata the $\mathrm{P} 2$ ' values range from $0.52-0.56$. It has been postulated that after remating there is intense sperm competition but there is no clear cut winner as sperm from both first and second males are used almost equally. In $D$. Pseudoananassae, however, sperm displacement could not be studied precisely due to lack of markers making it impossible to determine the paternity of progeny after remating. Nonetheless, in the three more closely related species of the complex too, the degree of sperm displacement was found to vary, establishing the fact that they have evolved different mechanisms during the process of speciation with regard to the pattern of sexual selection.

\section{Non Sexual Behaviour}

Studying non sexual behaviour such as oviposition site preference, larval pupation behaviour, feeding and locomotin etc. in closely related species may give significant insights into their adaptive divergence.
Oviposition site preference was studied in details in four species of Drosophila by Srivastava and Singh (1993 a, b), two of which were members of this complex D. bipectinata and D. malerkotliana. Females of all the species however, exhibited similar oviposition patterns, preferring peripheral area to central area of culture medium for oviposition. $D$. bipectinata and D. malerkotliana were also found to prefer the surface of the food medium rather than papers positioned vertically in the medium when given a choice (Srivastava and Singh, 2001). The effect of light condition, different chemicals in different concentrations and temperature on oviposition was also studied and it was found that oviposition is more under light condition in all the four species studied, the pattern is altered by different chemicals in high and low concentrations and egg laying is minimum at a low temperature of $19^{\circ} \mathrm{C}$ (Srivastava and Singh, 1996, 1997, 1998). Thus, D. bipectinata and D. malerkotliana were found to exhibit similar oviposition pattern that also altered comparably owing to different abiotic factors such as light conditions, chemicals and temperature. Therefore, the response of the two species to different circumstances does not differ much, with regard to oviposition patterns.

Pupation height is another important behaviour having implications on the successful eclosion of adult flies. The habitat of the fly has a lot of influence on the pupation of the larvae and the subsequent eclosion of fly from the puparium. In the laboratory however, the flies in most cases are raised on the same medium. In spite of this, pupation height varies in the members of this complex. D. bipectinata, D. parabipectinata and $D$. pseudoananassae pupate on the surface of food medium while $D$. malerkotliana pupates at the top of the culture bottle. On the surface too, pupation site varies among $D$. bipectinata, $D$. parabipectinata and D. pseudoananassae. While $D$. bipectinata pupates more on the centre, $D$. parabipectinata and $D$. pseudoananassae prefers the periphery for pupation. However, whether pupation would occur at the centre or periphery of the food medium also depends on the moisture content of the medium. Singh and Pandey (1991) found that while the mean pupation height in $D$. bipectinata ranged from 0.41 to $0.75 \mathrm{~mm}$, in D. malerkotliana it ranged from 1 to $1.3 \mathrm{~mm}$ (multiple strains of the two species were employed for this study). Thus, there is not only 
interspecific but also intraspecific variation in pupation height in this complex. $\mathrm{F}_{1}$ hybrids from reciprocal crosses were not found to differ in pupation site preference. Pandey and Singh (1993) also found that while there is no difference in pupation height between the males and females, it was found to be influenced strongly by moisture content of the food medium and density of the larvae. Pupation height was also found to be affected by abiotic factors such as light and temperature and biotic factors such as larval development time.

\section{Evolutionary Studies in the D. bipectinata Species Complex}

\section{Genetic Basis of Hybrid Male Sterility in the Complex}

The fact that interspecific hybridization is possible in this complex, given no choice situations in the laboratory, divulges interesting possibilities of doing evolutionary studies in the complex. Therefore, the intricacies of speciation may be exquisitely revealed through evolutionary studies involving interspecific hybridization. Among the interspecific hybrids in this complex, the females are fertile and the males are sterile and not surprisingly, the mechanism underlying hybrid male sterility captured the fancy of a couple of workers who studied it extensively. To begin with, the size of testes and seminal vesicles were compared across the four species by Mishra and Singh (2006a). The size of testes is a marker of the length of sperm and it was found to be similar in D. bipecinata, $D$. parabipectinata and D. pseudoananassae but significantly larger in D. pseudoananassae. In hybrid sons from crosses involving $D$. bipectinata, $D$. parabipectinata and D. malerkotliana the size of testes remained similar to parents, but in those from crosses involving $D$. pseudoananassae with the other three species, testes were found to be reduced and atrophied. However, it is known that hybrid sons from all crosses are sterile. Therefore, although small sized testes reflect sterility, normal sized ones do not necessarily confirm fertility. Similarly, the size of seminal vesicle was found to be comparable in $D$. bipectinata, $D$. parabipectinata and $D$. malerkotliana but significantly smaller in $D$. pseudoananassae. In hybrids from all crosses, the size of seminal vesicle was found to be uniformly reduced. Therefore, the size and morphology of seminal vesicles are better indicators of sterility than the testes. The severity of hybrid male sterility was also found to vary in hybrid males from different crosses. For example, in hybrids involving the three species $D$. bipectinata, $D$. parabipectinata and $D$. malerkotliana, the testes have sperm but they are not individualized and are immotile. However, hybrid males from crosses involving the three species with D. pseudoananassae have aspermic testes i.e. absence of sperm in their testes. Based on their analysis of morphology of testes and seminal vesicles, in the four species and their hybrids Mishra and Singh (2006a) concluded that D. bipectinata, D. parabipectinata and D. malerkotliana share a close phylogenetic relationship and D. pseudoananassae is distantly related to them.

Mishra and Singh (2006a), also found a strong asymmetry in testes phenotypes in reciprocal crosses of $D$. pseudoananassae with the other three species, with the testes size being markedly reduced in the sons from crosses where $D$. pseudoananassae was the female parent, than the respective reciprocal crosses. Therefore, it was postulated that incompatible interactions among the genetic factors on $D$. pseudoananassae $\mathrm{X}$ chromosomes and/or cytoplasmic factors and other factors (autosomal/ cytoplasmic) in the other species are involved in causing testes defects. Mishra and Singh (2005b), started with a preliminary study to determine the roles of $\mathrm{X}$ and $\mathrm{Y}$ chromosomes and their interactions with autosomes in hybrid male sterility in crosses between the three more closely related species, $D$. bipectinata, $D$. parabipectinata and D. malerkotliana. It was broadly postulated that hybrid sons of $D$. bipectinata $\times D$. parabipectinata are sterile due to incompatible interactions between $\mathrm{X}$-Y or X-autosomes or polygenes. For hybrid male sterility of sons of $D$. bipectinata $\times D$. malerkotliana and $D$. parabipectinata $\times D$. malerkotliana, $\mathrm{X}$-autosome interactions or polygenic interactions were pinpointed. Dissecting the genetic basis of hybrid male sterility further, Mishra and Singh (2006b), studied the role(s) of $\mathrm{Y}$ chromosome, cytoplasm and major sterility genes (MHS genes) in hybrid sterility. Also, by involving single recessive visible markers $X$-autosome and $\mathrm{X}-\mathrm{Y}$ interactions in hybrid sterility was studied (Mishra and Singh, 2007). It was found that interactions leading to hybrid sterility are not identical 
in different crosses. In $D$. bipectinata $\times D$. parabipectinata $\mathrm{X}-\mathrm{Y}$ interactions were implicated. In $D$. bipectinata $\times D$. malerkotliana and $D$. parabipectinata $\times D$. malerkotliana $\mathrm{X}$-autosomal interactions were found to be involved and in hybrids sons from crosses involving D. pseudoananassae, $\mathrm{X}-\mathrm{Y}$ interactions with high degree of incompatibility were accounted for.

\section{Degree of Crossability, Pattern of Mating Preference and Direction of Evolution}

Degrees of crossability among the members of this complex were first studied by Bock (1978). He found that $D$. bipectinata and D. parabipectinata were fully crossable and on the other hand, $D$. malerkotliana and D. pseudoananassae were barely crossable in either direction. Singh and Chatterjee (1991), found sexual isolation to be more or less uniform between sympatric and allopatric populations of $D$. bipecinata and $D$. malerkotliana, thereby proving that there is no character displacement for reproductive isolation between $D$. bipectinata and D. malerkotliana. Mishra and Singh (2006b) studied the degree of interspecific crossability in this complex. The degrees of crossability were found to be similar in reciprocal crosses except in the pair $D$. bipectinata-D. parabipectinata. When $D$. bipectinata was the female parent the degree of crossability was $96 \%$, whereas when $D$. parabipectinata was the female parent the degree of crossability was found to be $45 \%$. The degrees of crossability reduced drastically when $D$. pseudoananassae was involved. On average, degree of crossability was maximum between $D$. bipectinata-D. parabipectinata $(69 \%$, average crossability of reciprocals) and minimum between $D$. malerkotliana- D. pseudoananassae $3.5 \%$, average crossability of reciprocals).

The degree and pattern of sexual isolation among closely related species can be utilized for predicting the direction of evolution and deriving a phylogeny. Singh et al. (1981) studied sexual isolation among three species $D$. bipectinata, $D$. parabipectinata and D. malerkotliana in the complex by following the male choice method. $D$. pseudoananassae was not included in their study. They found deviation from randomness of mating in five out of six crosses, with the number of homogamic matings being far more than heterogamic matings. In only one of the crosses involving $D$. bipectinata and $D$. parabipectinata, females with $D$. parabipectinata males, difference between the number of homogamic and heterogamic matings was found to be insignificant. Watanabe and Kawanishi (1983) also studied sexual isolation in the complex and predicted the direction of evolution and geographic distribution based on the mode of mating preference of females. These studies were extended by Banerjee and Singh (2012) who did a more expansive study and involved $D$. pseudoananassae too. The direction of evolution among these species was discussed in the light of the model for asymmetric isolation proposed by Watanabe and Kawanishi (1979). The results suggested that while $D$. bipectinata, $D$. parabipectinata and D. malerkotliana are closely related to each other, $D$. pseodoananassae is distantly related to these three species, as it shows a strong symmetrical isolation with them. The species pairs $D$. bipectinata - D. parabipectinata and $D$. parabipectinata - D. malerkotliana were found to exhibit asymmetrical sexual isolation. Based on this, it was suggested that D. bipectinata and $D$. malerkotliana share a common ancestor where as $D$. parabipectinata has been derived from $D$. bipectinata.

Banerjee and Singh (2014) also tested the mating preference of hybrid females towards parental males. Pattern of mating preference of hybrid daughters derived from reciprocal interspecific crosses involving $D$. bipectinata, D. parabipectinata and $D$. malerkotliana was found to be similar providing evidence that mate recognition in these species has no influence of the sex of parental species. In other words, the genes responsible for mate recognition do not show any maternal inheritance. Also, the daughters from reciprocal crosses among these species prefer one of the parental males better over the other indicating that the genome of one of the parental species is dominant over the other. The daughters of $D$. parabipectinata and $D$ malerkotliana were found to have poor receptivity, as they mated infrequently with both the parental males, worse with D. malerkotliana males. This indicates that though fertile, their mate recognition system is affected severely due to hybridization. Consistent with earlier findings, it was very difficult to hybridize $D$. 
pseudoananassae with the other three species of the complex. When D. pseudoananassae was the male parent, getting progeny turned out to be all the more difficult. Thus, it was postulated that the courtship rituals of $D$. pseudoananassae has diverged to such an extent that the females of the other three species recognize it with difficulty. It may also be possible that the females have evolved a hostile reproductive tract environment, killing sperm from the males of a distantly related species like $D$. pseudoananassae.

Daughters of $D$. bipectinata females $\times D$. pseudoananassae males were found to mate more with $D$. bipectinata males than the daughters of the other reciprocal cross, indicating that when $D$. pseudoananassae is involved the pattern of mating preference of hybrid daughters is very different. The possible explanation for this can be that there is some maternal effect associated with $D$. pseudoananassae females which does not allow daughters of $D$. pseudoananassae females $\times D$. bipectinata males to recognize $D$. bipectinata males Daughters of $D$. parabipectinata females $\times D$. pseudoananassae males were found to mate more with $D$. pseudoananassae males than the daughters of the other reciprocal cross. Thus, the maternal effect associated with $D$. pseudoananassae females does not play any role here and this preference pattern cannot be explained with any possible hypothesis and needs further investigations.

\section{Studies on Fluctuating Asymmetry (FA)}

Fluctuating asymmetry (FA) is defined as subtle deviations from perfect bilateral symmetry, evident in differences between the right and the left sides of any given trait. It is a pattern of variation between sides and measures developmental instability (Palmer 1994). Differences in the level of FA may be used for comparing developmental precision among closely related species and thus may give an idea whether developmental stability was affected during the divergence and separation of populations into distinct species. Banerjee and Singh (2015a) compared the level of FA in certain morphological traits in the four members of the complex. The magnitude of FA was found to vary significantly among traits and the composite fluctuating asymmetry (CFA) values were found to be higher for males than females in all the four species. It was concluded that FA shows trait specific variations and males are more prone to developmental perturbations. However, the FA levels remain more or less comparable in the four species proving that developmental precision remains same in the four species irrespective of the evolutionary divergence reached among them. However, since they tested FA in the laboratory populations, it was postulated that the level of FA may have come to match each other in the four species owing to inbreeding as laboratory populations are maintained by transferring small number of flies in each generation. Inbreeding is known to affect the size of morphometric traits. For example, Trehan and Gill (1986) have demonstrated that wing morphology is affected in D. malerkotliana due to inbreeding.

Further, the level of FA in different morphological traits was also scored in interspecific hybrids and compared with that of parental species with the idea that it would throw light on the degree of divergence between the parental species. If they have not diverged much, the interspecific hybrids may have a similar FA level, incompatibilities between their genomes being negligible. On the other hand, if there is substantial divergence, the level of FA may be higher due to incompatibility between the genomes of the parental species. However, except in a few cases, no significant differences could be detected in the level of FA in hybrids as compared to pure species (Banerjee and Singh, 2015b). However, a number of other abnormalities could be detected in the progeny from some of the crosses. In the crosses $D$. pseudoananassae females $\times D$. bipectinata males and $D$. pseudoananassae females $\times D$. parabipectinata males, the hybrid sons were found to have severely compromised eye symmetry and poor viability (Singh and Banerjee, 2015), and in crosses involving $D$. pseudoananassae as one of the parental species, most of the hybrid daughters were found to have dystrophied ovaries. Right from the very recognition of this complex, it has always been claimed that the hybrid males are sterile, but females are fertile. Yet, females with dystrophied ovaries cannot be fertile, so there must be some degree of sterility in the hybrid females too. This was investigated and it was found that indeed fertility is drastically reduced in hybrid females from all crosses save ones involving $D$. bipectinata $\times D$. parabipectinata and $D$. bipectinata $\times D$. 
malerkotliana (Banerjee and Singh, 2015c). Hence it was concluded that specific developmental pathways are more susceptible to developmental disturbances due to genomic incompatibilities than the large complex system bringing about developmental stability.

Polak et al. (2004) studied variation in sex comb, hailed as a sexual ornament in different populations of $D$. bipectinata. The magnitude of sex comb was said to be undergoing adaptive diversification. They divided the comb into three segments $\mathrm{C} 1, \mathrm{C} 2$ (the two rows in the first tarsal segment) and C3 (in the second tarsal segment). Significant sexual selection was detected for increasing the number of teeth in $\mathrm{C} 2$ and body size and simultaneously for reducing comb positional fluctuating asymmetry. On the other hand, sexual selection was not detected either for increasing the size of other comb segments or decreasing the FA therein. A significant positive genetic correlation was also detected between the magnitude of this sexual trait and its positional fluctuating asymmetry indicating that counteracting and independent selection pressures maintain genetic variation (necessary for adaptive diversification) while at the same time sustaining sexual selection. The developmental instability (DI)-sexual selection hypothesis says that large size and symmetry in secondary sexual traits are favoured by sexual selection. However, the findings of the most recent work by Polak et al. (2015), on D. bipectinata sex combs are inconsistent with the DI-sexual selection hypothesis. FA-size correlations were significantly positive in field caught males from five geographic localities. However, after correcting for trait size, FA was significantly negatively correlated with body size indicating that FA in comb size reflects an individual genetic quality and form independent units of the genome.

\section{Future Implications}

The annals of studies done on the D. bipectinata species complex are immense. However, a perusal through the archives reveals that every aspect has not been unearthed. There are many studies in which only one species, mostly $D$. bipectinata or $D$. malerkotliana has been usedmore like a model for understanding a phenomenon. However, the species and subspecies of the bipectinta complex in addition to serving as independent models also turns out to be a wonderful model of a single complex. Studying the various aspects of behaviour and population genetics elaborately in the four species and comparisons may help solve many puzzles regarding the paths taken during speciation. While a number of studies have been done, there are still many gaps which need filling. A number of population genetical studies with regard to intra and interspecific chromosomal polymorphisms have been done but the results are not consistent throughout. Especially, Bock (1971 b) and Tomimura et al. (2005) predict different phylogenies based on their chromosomal studies. This is surprising because the differences between species ought to be fixed (during a short evolutionary time scale) (Patterson and Stone 1952).

Studies on nucleotide divergence are not sufficient either and there is still much to be done. From the basic and straightforward investigations on nucleotide divergence, research should evolve into functional genomics studies in this complex. For example, comparisons of the expression profiles (tissue wise) among the species and between parental species and their hybrids have remained untouched in this complex. The genomic studies may also be extended to transcriptomics or expression studies. In fact, Signor et al. (2013) generated genomic resources by utilizing the transcriptome sequence data of a number of species of the ananassae subgroup and the species of this complex have also been included. The generation of gargantuan data from such a study and interpretation of the data would require huge investments both in terms of time and money, but the results would indeed be very interesting and open up newer prospects.

As regards behavioural studies, though courtship behaviour has been explored sufficiently throwing light on the interspecific differences among the species yet there are many aspects which need expansion. Studying the courtship patterns of the interspecific hybrid male will be very valuable in understanding the mode of inheritance of the courtship rituals. Since, the preference pattern of interspecific hybrid females with parental males have already been studied, studies on the preference pattern of the hybrid females with hybrid males together with the courtship rituals of the hybrid males would offer interesting glimpses into the relationship shared by different species pair in this 
complex. This would be an amalgam of behavioural and evolutionary studies and would be able to cover many gaps.

Though the genetic basis of hybrid male sterility has been studied to a large extent, there is a need of further analyses as only the gross roles of Xchromosome, autosomes or major sterility genes have been identified. For digging deeper, one needs to have more markers. There is a need therefore to generate more mutant markers for which molecular methods such as site directed mutagenesis may be used. Markers (preferably visible mutant markers) in $D$. pseudoananassae, would also aid in studying sperm displacement in this species. It is the only species in this complex in which the phenomenon has not been studied and studying the same would allow a wholesome investigation into how sperm displacement

\section{References}

Aotsuka T and Tobari Y N (1983) Isozyme variations, morphological divergence and reproductive isolations in the Drosophila bipectinata species complex. In: The overseas scientific expedition for the collection of Drosophilid flies, 1971-1983 (Ed: Kitagawa O) pp 62-72, The Ministry of Education, Science, and Culture of Japan, Tokyo, Japan

Banerjee P and Singh B N (2012) Interspecific sexual isolation and phylogeny among different members of the Drosophila bipectinata species complex Genetica $14075-85$

Banerjee P and Singh B N (2014) Pattern of mating preference of interspecific hybrid females and phylogeny in the Drosophila bipectinata species complex J Genet 93 495499

Banerjee P and Singh B N (2015a) Studies on fluctuating asymmetry (FA) for certain morphological traits in four species of the Drosophila bipectinata complex Genetika 47 535-548

Banerjee P and Singh B N (2015b) Interspecific hybridization does not affect the level of fluctuating asymmetry (FA) in the D. bipectinata species complex Genetica 143 459-471

Banerjee P and Singh B N (2015c) The Drosophila bipectinata species complex: degree of sterility and dystrophied ovaries in interspecific hybrid females $J$ Genet in press

Banerjee R and Singh B N (1996) Inversion polymorphism in natural populations of Drosophila bipectinata Cytobios patterns may vary among closely related species.

Also, molecular evolutionary studies in this complex performed with the newest techniques would definitely lead us to interesting breakthroughs and will refine our knowledge about speciation. Therefore, we are very hopeful that the future would see this promising complex come more to the fore and better our understanding of speciation.

\section{Acknowledgements}

Financial assistance in the form UGC-BSR Faculty Fellowship award to B N S and Meritorious Fellowship to $\mathrm{PB}$ is gratefully acknowledged. We also thank the anonymous reviewer for his/her helpful comments that greatly improved the review.

\section{$8731-43$}

Banerjee R and Singh B N (1998) Evidence for coadaptation in geographic populations of Drosophila bipectinata J Zool Syst Evol Res 36 1-6

Bock I R (1971a) Taxonomy of the Drosophila bipectinata species complex Univ Tex Publ 6 273-280

Bock I R (1971b) Intra and interspecific chromosomal inversions in the Drosophila bipectinata species complex Chromosoma 34 206-229

Bock I R (1978) The bipectinata complex: a study in interspecific hybridization in the genus Drosophila (Insecta: Diptera) Aust J Biol Sci 31 197-208

Bock I R and Wheeler M R (1972) The Drosophila melanogaster species group Univ Tex Publ 7213 1-102

Crossely S A (1986) Courtship sound and behaviour in the four species of the Drosophila bipectinata complex Anim Behav 34 1146-1159

Crossley S A and Bennet- Clark H C (1993) The response of Drosophila parabipectinata to stimulated courtship songs Anim Behav 45 559-570

Das A and Singh B N (1992) Heterosis associated with chromosomal inversions in Drosophila bipectinata Kor J Genet 14 173-178

Duda O (1923) Die Orientalischen und australischen Drosophiliden-Arten (Dipteran)des ungarischen national museum zu Budapest Ann Mus Nat Hung 20 24-59

Ewing A W and Bennet-Clark H C (1968) Courtship songs of 
Drosophila Behaviour 31 288-301

Gupta J P and Panigrahy K K (1990) Chromosomal polymorphism in Indian populations of Drosophila bipectinata Duda Genetica 82 45-49

Gupta J P, Aotsuka T, Inaba A and Kitagawa O (1993) Analysis of mt DNA in two species of the bipectinata species complex of Drosophila Jpn J Genet $\mathbf{6 8} 257-264$

Gupta J P, Dwivedi Y N and Singh B K (1980) Natural hybridization in Drosophila Experientia 36 290-291

Hegde S N and Krishna M S (1996) Effect of bottlenecks on incipient sexual isolation mating activity and fertility in Drosophila malerkotliana Ind J Exp Biol 34 440-443

Hegde S N and Krishna M S (1997) Size-assortative mating in Drosophila malerkotliana Anim Behav 54 419-426

Hegde S N and Krishna M S (1999) Bottleneck effect on intraand interspecific competition in Drosophila malerkotliana Ind J Exp Biol 37 359-364

Hegde S N and Krishnamurthy N B (1976) Studies on the genetics of isozymes in the hybrids of Drsophila bipectinata complex Proc Dunn Dobzh Symp Genet 90-97

Hegde S N and Krishnamurthy N B (1979) Studies on mating behaviour in Drosophila bipectinata complex Aust J Zool 27 421-431

Hegde S N and Naseerulla M K (1992) Correlative studies on mating speed and metric traits in Drosophila malerkotliana Ind J Exp Biol 30 334-338

Hegde S N Naseerulla M K and Krishna M S (2000) Variability of morphological traits in Drosophila bipectinata complex Ind J Exp Biol 38 797-806

Jha A P and Rahman S M Z (1972) Cytogenetics of natural populations of Drosophila I Role of chromosomal inversions in the evolution of the Drosophila bipectinata species complex Chromosoma 37 445-454

Jha A P, Rahman S M Z (1973) On crossing between Drosophila bipectinata and D. malerkotliana Cytologia 38 425-436

Jha A P, Mishra D N and and Pandey B N (1979) Alcohol dehydrogenase isoenzymes in Drosophila species hybrids Ind J Exp Biol 17 647-649

Jha A P, Mishra D N and Pandey B N (1978) Silent alleles for alcohol dehydrogenase in Drosophila malerkotliana. Cell chromosome newslet 11 97-81

Jha A P, Pandey B N and Korochkin L T (1980) Comparative study of alcohol dehydrogenase substrate specificities in Drosophila malerkotliana Ind J Exp Biol 18 482-485

Kamimura Y and Polak M (2010) Does surgical manipulation of Drosophila intromittent organs affect insemination success

\section{Proc R Soc B278 815-816}

Kopp A and True J R (2002) Phylogeny of oriental Drosophila melanogaster species group: a multilocus reconstruction Syst Biol 51 786-805

Kopp A and Barmina O (2005) Evolutionary history of the Drosophila bipectinata species complex Genet Res Camb 85 23-46

Kopp A and Frank A K (2005) Speciation in progress? A continuum of reproductive isolation in Drosophila bipectinata Genetica 125 55-68

Kopp A, Frank A K and Barmina O (2006) Interspecific divergence, intra chromosomal recombination and phylogenetic utility of Y-chromosomal genes in Drosophila Mol Phylogenet Evol 38 731-741

Krishna M S and Hegde S N (1996) Bottleneck effect on sexual isolation in chromosomally monomorphic and polymorphic populations of Drosophila malerkotliana Cell Chromosome Res 191 1-8

Krishna M S and Hegde S N (1997) Body size, mating success and advantage of large flies in Drosophila bipectinata species complex Ind J Exp Biol 35 1341-1347

Krishna M S, Snatosh H T and Hegde S N (2012) Offspring of older males are superior in D. bipectinata Zool Stud $\mathbf{5 1} 72$ 84

Lachaise D, Cariou M L, David J R, Lemeunier F, Tsacas L and Ashburner M (1988) Historical biogeography of the Drosophila melanogaster species subgroup J Evol Biol 22 $159-225$

Lemeunier F, David J R and Tsacas L (1986) The melanogaster species group. In The genetics and biology of Drosophila (Eds Ashburner M, Carson H L, and Thompson J N)pp 147-256 Academic Press, London, UK

Matsuda M, Tomimura Y and Tobari Y N (2005) Reproductive isolation among geographic populations of Drosophila bipectinata Duda (Diptera, Drosophilidae) with recognition of three subspecies Genetica 125 69-78

Markow T A and O'Grady P M (2005) Drosophila: a Guide to Species Identi?cation and Use. Elsevier, London

Matsuda M, Ng C S, Doi M Kopp A and Tobari Y N (2009) Evolution in Drosophila ananassae species subgroup Fly (Austin) 3 157-169

Mishra P K and Singh B N (2005 a) Why hybrid males are sterile in Drosophila? Curr Sci 89 1823-1819

Mishra P K and Singh B N (2005b) Genetic basis of hybrid male sterility among three closely related species of Drosophila Ind J Exp Biol 43 455-461 
Mishra P K and Singh B N (2006a) Drosophila bipectinata species complex: study of phylogenetic relationship among four members through analysis of morphology of testes and seminal vesicles J Zool Syst Evol Res 44 175-179

Mishra P K and Singh B N (2006b) Genetic interactions underlying hybrid male sterility in the Drosophila bipectinata species complex Genes and Genet Syst 81 193-200

Mishra P K and Singh B N (2007) Assessing the putative roles of $\mathrm{X}$-autosome and $\mathrm{X}-\mathrm{Y}$ interactions in hybrid male sterility of Drosophila bipectinata species complex Genome $\mathbf{5 0}$ 653-659

Narda R D (1968) Genetical and isolating mechanisms in the ananassae subgroup (Sophophora Drosophila)VI Cytological divergence in Drosophila ananassae, Drosophila bipectinata and Drosophila malerkotliana Caryologia 21 293-301

Naseerulla M K and Hegde S N (1993a) Lack of correlation between mating activity and Est-1 polymorphism in three natural and laboratory populations of $D$. bipectinata Ind $J$ Exp Biol 31 215-218

Naseerulla M K and Hegde S N (1993b) Microclimate variations at two enzyme loi in $D$. malerkotliana and $D$. bipectinata Dros inf Serv 72 159-161

Naserulla M K and Hegde S N (1993c) A new inversion in Drosophila maerkotliana from Varanasi, India Dros infom Ser 72158

Ohtsuka C, Aotsuka T and Tobari Y N (1983) Mating behaviour of Drosophila bipectinata complex. In the overseas scientific expedition for the collection of Drosophila flies1971-1983. Edited by O. Kitagawa. The Ministry of Education, Science, and Culture of Japan, Tokyo, Japan pp.73-74

Parshad R and Paika I J (1964) Drosophilid survey of India. II Taxonomy and cytology of the subgenus Sophophora (Drosophila) Res Bull Punj Univ 15 222-252

Pandey M B and Singh B N (1992) Pupation height of $F_{1}$ hybrids obtained from reciprocal crosses between Drosophila bipectinata and Drosophila malerkotliana Dros Inf Serv $\mathbf{7 1} 257-259$

Pandey M B and Singh B N (1993) Effect of biotic and abiotic factors on pupation height in four species of Drosophila Ind J Exp Biol 31 912-917

Polak M, Starmer WT Wolf LL (2004) Sexual selection for size and symmetry in diversifying secondary sexual character in Drosophila bipectinata Duda (Diptera:Drosophilidae) Evolution 58 597-607

Polak M and Simmon L W (2009) Secondary sexual trait size reveals competitive fertilization success in Drosophila bipectinata Duda Behav Ecol 20 753-760

Polak M, Hooker K J and Tyler F (2015) Consistent positive covariation between fluctuating asymmetry and sexual trait size: a challenge to the developmental stability-sexual selection hypothesis Symmetry 7 976-993

Parkash R (1993) Biochemical differences among three acid phosphatase allozymes in Drosophila malerkotliana Dros Inf Serv 72 117-118

Parkash R, Sharma S and Sharma M (1994) Patterns of starvation and desiccation tolerance in Drosophila bipectinata and Drosophila makerkotliana Biol Entl Bl 113 355-364

Santosh H T and Krishna M S (2013) Relationship between male age, accessory gland, sperm transferred, and fitness traits in Drosophila bipectinata J Insect Sci 13 159-172

Sharma M, Sharma S and Parkash R (1993a) ADH polymorphism and ethanol tolerance in three species of ananassae species subgroup Evol Biol 7 51-62

Sharma S, Parkash R and Sharma M (1993b) Ethanol resourse utilization in Drosophila melanogaster and Drosophila bipectinata Natl Acad Sci Lett 16 259-262

Sharma S, Parkash R and Sharma M (1994) Geographical differentiation of allozyme polymorphism and ethanol tolerance in Drosophila malerkotliana populations from India Biol Zentl Bl 113 365-378

Shivakumar P and Krishna M S (2014) Male age influence on male remating and progeny production in Drosophila malerkotliana American J Biosci 2 101-107

Signor S, Seher T and Kopp A (2013) Genomic resources for multiple species in the Drosophila ananassae species subgroup Fly 7 48-57

Singh B N (2015) Species and genetic diversity in the genus Drosophila inhabiting the Indian subcontinent J Genet 94 351-361

Singh B N, Dwivedi Y N and Gupta J P (1981) Sexual isolation among three species of the Drosophila bipectinta species complex Ind J Exp Biol 19 898-900

Singh B N and Chatterjee S (1991) No character displacement for reproductive isolation between Drosophila bipectinata and Drosophila malerkotliana Genome 34 849-852

Singh B N and Chatterjee S (1991) Evidence for incipient sexual isolation within Drosophila bipectinata Evol Biol 5 105113

Singh B N and Chatterjee S (1992) Intraspecific sexual isolation in Drosophila Ind J Exp Biol 30 260-263

Singh B N and Das A (1991) Linkage disequilibrium between 
inversions in Drosophila bipectinata Biol Zentbl 110157 162

Singh B N and Lata S (1992) Interspecies variation in the number of sternopleural bristles in Drosophila Kor J Genet $\mathbf{1 4}$ 145-151

Singh B N and Pandey M (1991) Intra- and interspecies variations in pupation height in Drosophila Ind J Exp Biol 29 926929

Singh B N, Singh O P and Gupta J P (1996) The antennae and mating behaviour of Drosophila females Experientia $\mathbf{3 8}$ 237-238

Singh B N and Banerjee R (1995) Chromosomal variability and interracial hybridization in Drosophila bipectinata Cytobios 82 219-227

Singh B N and Banerjee R (1997) Increase in degree of inversion polymorphism in Drosophila bipectinata populations transferred to laboratory conditions J Zool Syst Evol Res 35 153-157

Singh B N and Sisodia S (1996a) No effect of cut wing mutation on mating propensity in Drosophila bipectinata Biol Zentbl $11546-50$

Singh B N and Sisodia S (1996b) Evidence for positive assortative mating within Drosophila bipectinata Curr Sci 71517 518

Singh B N and Sisodia S (1997) Evidence for rare male mating advantage in Drosophila bipectinata Genetika 29 69-81

Singh B N and Sisodia S (1999) Mating propensity in $D$. bipectinata under different sex ratios and choice situations Curr Sci 76 222-225

Singh S R, Singh B N and Hoenigsberg HF (2002) Female remating, sperm competition and sexual selection in Drosophila Genet and Mol Res 1 178-2002

Singh B N, Singh A and Banerjee P (2013) Persistence of an inversion in laboratory stocks of $D$. bipectinata Dros Inf Ser 96 1-3

Singh A and Singh B N (2013) Studies on remating behaviour in the Drosophila bipectinata species complex: intra- and interspecific variations Behav Proc 113 105-109

Singh A and Singh B N (2014a) Mating latency, duration of copulation and fertility in four species of the Drosophila bipectinata complex Ind J Exp Biol 52 175-180

Singh A and Singh B N (2014b) Studies on remating behaviour in the Drosophila bipectinata species complex. Evidence for sperm displacement in Drosophila bipectinata Curr Sci 107 511-513

Singh A and Singh B N (2015) Sperm displacement in the Drosophila bipectinata species complex: evidence for interspecific variations Behave Proc 113 105-109

Singh B N and Banerjee P (2015) Sex specific asymmetry in development of eyes in the Dtosophila bipectinata species complex J Genet 94 493-495

Sisodia S and Singh B N (1996a) Mating propensity of interstrain hybrids in Drosophila bipectinata Braz J Genetics 19365368

Sisodia S and Singh B N (1996b) Evidence for positive correlation between duration of copulation and fertility in Drosophila bipectinata Zool Stud 35 25-29

Somashekar K, Krishna MS, Hegde SN and Jayaramu SC (2011) Effect of age on female reproductive success in Drosophila bipectinata J Insect Sci 11 132-148

Srivastava T and Singh B N (1993a) Intraspecific variation with respect to oviposition site preference in certain Indian species of Drosophila Evol Biol 7 193-205

Srivastava T and Singh B N (1993b) Oviposition site preference in four species of Drosophila Ind J Exp Biol 31 460-462

Srivastava T and Singh B N (1996) Rhythmicity in oviposition pattern in light and darkness in four Indian species of Drosophila Biol Res 29 355-360

Srivastava T and Singh B N (1997) Effect of different chemicals on oviposition pattern in four Indian species of Drosophila Rev Brasil Biol 57 571-577

Srivastava T and Singh B N (1998) Effect of temperature on oviposition in four species of the melanogaster group of Drosophila Rev Brasil Biol 58 489-493

Srivastava T and Singh B N (2001) Choice of oviposition site between surface of the medium and paper in four Indian species of Drosophila Ind J Exp Biol 39 383-386

True J R (2003) Insect melanism: the molecules matter. Trends Ecol Evol 18 640-647.

Tomimura Y, Matsuda M, and Tobari Y N (2005) Chromosomal phylogeny and geographical divergence in the Drosophila bipectinata complex Genome 48 487-502

Trehan K S and Gill K S (1986) Effect of inbreeding on wing morphology in Drosophila malerkotliana Dros Inf Serv 63130

Watanabe T K and Kawanishi M (1979) Mating Preference and the direction of evolution in Drosophila Science 205 906907

Watanabe T K and Kawanishi M (1983) Stasipatric speciation in Drosophila Jpn J Genet $\mathbf{5 8} 269$

Yang S Y, Wheeler L and Bock I R (1972) Isozyme variations and phylogenetic relationships in Drosophila bipectinata species complex Univ Texas Publ 7213 213-227. 
